\title{
Dramatic response of refractory sarcoidosis under ruxolitinib in a patient with associated JAK2-mutated polycythemia
}

\author{
To the Editor:
}

Sarcoidosis is a systemic disorder of unknown aetiology characterised by the formation of non-caseating epithelioid granulomas in involved organs. Although a spontaneous remission is frequent, up to $28-53 \%$ of patients may follow a persistent active course for more than 5 years $[1,2]$. Outcome may be severe in a subset of these patients. Sarcoidosis treatment relies on three lines of therapies respectively corticosteroids, immunosuppressive medications and anti-tumour necrosis factor- $\alpha$ (anti-TNF- $\alpha$ ) therapy according to severity.

We report here the case of a woman of European ancestry, born in 1958, diagnosed in 1999 with a multivisceral sarcoidosis. The institutional review board for human studies approved this study; and written informed consent was signed by the patient. At diagnosis work-up, the patient suffered from facial unsightly cutaneous lesions and diffuse pulmonary involvement with moderate pulmonary fibrosis on high resolution computed tomography. Skin biopsy evidenced typical non-caseating granulomas. First-line therapy consisted of oral prednisone (1 mg per $\mathrm{kg}$ of body weight each day) with a good clinical response. Prednisone doses were tapered and stopped after 3 years of treatment. Then a 2-year course of hydroxychloroquine was marked by a relapse with skin and lung involvement, progression and occurrence of a specific nasosinusal localisation. Several treatments were successively initiated (methotrexate, azathioprine, leflunomide, infliximab and ultimately adalimumab) but had to be stopped following either no efficacy or adverse effects. Prednisone was then resumed alone without any adverse effect. Minimal doses needed for controlling sarcoidosis gradually increased over time from 2006 to 2016 , from $8 \mathrm{mg} \cdot \mathrm{day}^{-1}$ of prednisone to more than $20 \mathrm{mg} \cdot \mathrm{day}^{-1}$ as she experienced with this dosage a new sarcoidosis flare at skin, and both nasosinusal and pulmonary levels. Figure 1a illustrates the evolution over time of corticosteroid doses given for controlling sarcoidosis.

In February 2017, she was diagnosed with polycythaemia vera (haemoglobin $18.3 \mathrm{~g} \cdot \mathrm{dL}^{-1}$; human T-cell $58.8 \%$; red cell mass $31 \%$ ) with positive blood V617F JAK2 mutation detected in the blood. A course of hydroxyurea had to be rapidly interrupted after a couple of weeks because of drug secondary side-effect (hepatic intolerance with cholestatic hepatitis). Rather than interferon- $\alpha$, considered as non-desirable in the context of a non-controlled granulomatosis process, ruxolitinib, a potent and selective ATP-competitive inhibitor of JAK1 and JAK2 kinases, was initiated on June 26, 2017 (5 mg orally every $12 \mathrm{~h}$ ). 3 months after ruxolitinib introduction, skin lesions disappeared ad integrum, lung infiltration dramatically decreased with only persistent irreversible fibrotic lesions (figure $1 \mathrm{~b}$ ) and pulmonary function tests dramatically improved. Vital capacity went from $1.63 \mathrm{~L}$ ( $66 \%$ of predictive value) before ruxolitinib to $2.49 \mathrm{~L}\left(+36 \%\right.$ of predicted value) after 6 months. Prednisone could be tapered to $10 \mathrm{mg} \cdot \mathrm{day}^{-1}$ after 6 months of ruxolitinib without any further disease relapse, as confirmed on computed tomography and

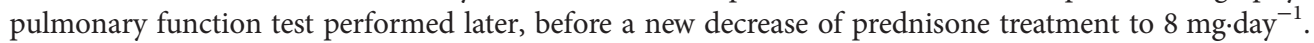

This report concerns a case of longstanding (18 years) sarcoidosis characterised by a multivisceral flare and increased corticosteroids needs with a rapid and dramatic response following initiation of ruxolitinib, a potent and selective ATP-competitive inhibitor of JAK1 and JAK2 kinases against myeloproliferative neoplasms [3], for treating a concomitant polycythemia vera.

@ERSpublications

Potential role of the JAK-STAT pathway in sarcoidosis

http://ow.ly/L8Du30m78XI

Cite this article as: Rotenberg C, Besnard V, Brillet P-Y, et al. Dramatic response of refractory sarcoidosis under ruxolitinib in a patient with associated JAK2-mutated polycythemia. Eur Respir J 2018; 52: 1801482 [https://doi.org/10.1183/13993003.01482-2018]. 

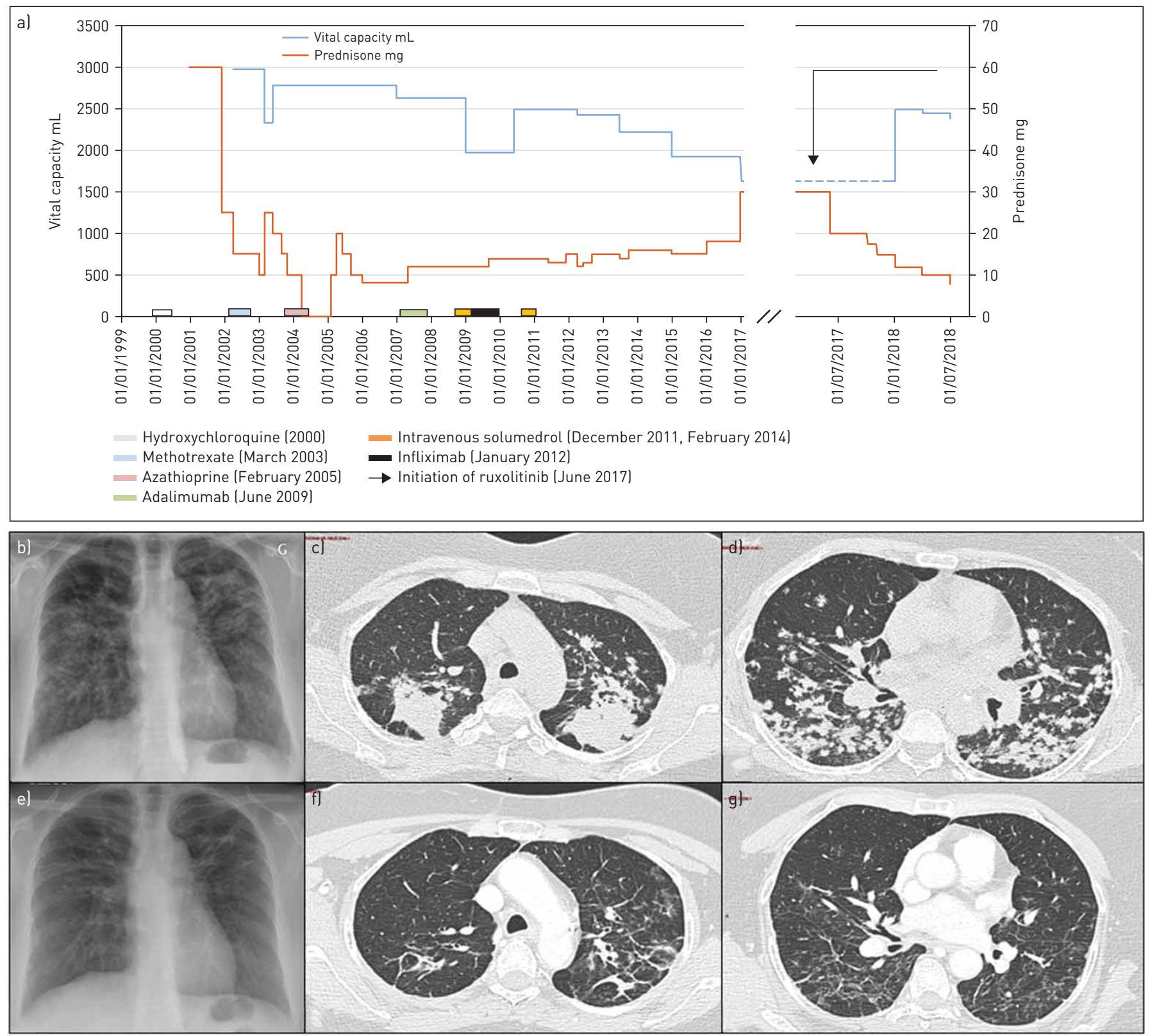

FIGURE 1 Patient evolution before and after ruxolitinib treatment. a) Evolution of vital capacity (blue dotted line) and prednisone treatment (brown line) during follow-up. Chest radiograph $(b, e)$ and computed tomography images ( $c, d$ and $f, g)$ : before ruxolitinib initiation (b-d) and 6 -months (e) or 9-months (f, g) after, showing improvement of nodular opacities and consolidation with residual linear opacities.

The JAK (Janus kinase) family of cytoplasmic tyrosine kinases JAK1, JAK2, JAK3 and TYK2 (tyrosine kinase 2) is required for signalling by cytokine and growth factor receptors that lack intrinsic kinase activity. Dysfunctions in the JAK/STAT pathway are implicated in various autoimmune diseases, including rheumatoid arthritis, psoriasis and inflammatory bowel diseases [4]. JAK2 acts as a downstream mediator of various cytokines and growth factors, including IL (interleukin)-3, IL-6, interferon- $\gamma$, granulocyte-macrophage colony-stimulating factor, thrombopoietin and erythropoietin [5]. The JAK/STAT pathway was recently incriminated in the pathophysiology of sarcoidosis [6-8]. In a genome-wide peripheral blood gene expression analysis, T. Zhou and co-workers showed that in addition to the T-cell receptor (TCR) signalling, the JAK/STAT signalling pathway was the most significantly represented pathway in sarcoidosis [9] and was associated with a significant trend in the severity score [10].

Ruxolitinib is the first-in-class drug FDA-approved for the treatment of high and intermediate-risk myelofibrosis in first line therapy and is also approved in polycythemia vera as a second-line therapy. Ruxolitinib effects are correlated with decreased levels of phosphorylated JAK1 and JAK2 and of signal 
transducer and activator of transcription 5 and 3 (STAT5 and STAT3). In addition to its known effect on the erythroid lineage, ruxolitinib can reduce the JAK/STAT signalling by decreasing phosphorylated STAT3 (pSTAT3) levels in peripheral blood leukocytes [11]. JAK inhibition induced by ruxolitinib treatment has been demonstrated to modify/reduce several immunologic mechanisms implicated in the development of granulomatous inflammation in sarcoidosis including expression of pro-inflammatory mediators IL- 6 and TNF- $\alpha$, microRNA involved in regulatory pathways including transforming growth factor- $\beta$ signalling, MAPK (mitogen-activated protein kinases) signalling, mTOR (mammalian target of rapamycin) signalling, and CXCR4 expression [12, 13]. Ruxolitinib was successfully used to treat autoimmune cytopenias and chronic mucocutaneous candidiasis [14]. Ruxolitinib reverses dysregulated T-helper cell responses and controls autoimmunity caused by a novel STAT1 gain-of-function mutation and cutaneous lupus erythematosus [15]. Ruxolitinib inhibits the expression of cytokines characteristic of cutaneous lupus erythematosus. The improvement was linked to a reduced hyperresponsiveness to type I and II interferons, normalised $\mathrm{T}_{\mathrm{H}} 1$ and follicular $\mathrm{T}$-helper cell responses and a better $\mathrm{T}_{\mathrm{H}} 17$ differentiation. Altogether, these data suggest that ruxolitinib may have participated in sarcoidosis improvement by modulating both immune and inflammatory responses.

While glucocorticoids (GC) are the most commonly used medication for the treatment of sarcoidosis, GC resistance is widely recognised as a complicating factor in the management of sarcoidosis leading to the recourse of alternative therapies as immunosuppressive (methotrexate, azathioprine, etc.) or anti-TNF- $\alpha$ biotherapy (infliximab or adalimumab). A link between GC resistance and the JAK/STAT signalling pathway was previously shown with the formation of glucocorticoid receptor (GCR) GCR/STAT5 heterodimers. GC responsiveness of genes containing GC response element (GCRE) in their promoters was diminished in presence of GCR/STAT5 complexes [16]. Formation of the GCR/STAT5 inhibited the nuclear import of GCR, reducing its transcriptional activation on GCRE containing genes and thus triggering steroid insensitivity [17]. Likewise, JAK inhibition induced by ruxolitinib or other inhibitors of the IL-7 receptor/JAK/STAT signalling has been described to enhance the efficacy of GCs in specific subset of T-cell acute lymphoblastic leukemia by modulating the expression of genes involved in apoptosis [18]. Notably, Delgado-Martin et al. [18] showed that GCs downregulated mRNA levels of pro-survival $B C L 2$ and upregulated pro-apoptotic BIM in lymphoblasts. These data suggest that in the present case report, the need for an increase in the dose of corticosteroids during the course of sarcoidosis might has been linked with the progressive expression of the V617F JAK2 mutation in polycythemia vera. Strikingly, introduction of ruxolitinib drastically improved pulmonary function and reduced skin lesions, allowing a major reduction in the corticosteroid treatment.

The JAK/STAT signalling pathway is a new step for clinical and therapeutic advances in sarcoidosis. Further investigations are needed to determine their risk/benefit ratio and selection of the most appropriate patients for therapy targeting this pathway. Whether or not anti-JAK therapy might be a recourse in multiresistant severe sarcoidosis without associated polycythemia needs to be clarified before considering it as a new candidate for treatment in difficult sarcoidosis.

Cécile Rotenberg ${ }^{1,5}$, Valérie Besnard ${ }^{2,5}$, Pierre-Yves Brillet $^{3}$, Stéphane Giraudier ${ }^{4}$, Hilario Nunes ${ }^{1,2}$ and Dominique Valeyre ${ }^{1,2}$

${ }^{1}$ Dept of Respiratory Diseases, APHP, Avicenne Hospital, Bobigny, France. ${ }^{2}$ EA 2363 "Hypoxia \& the lung: fibrosing pneumonitis, ventilatory and circulatory modulations", UFR SMBH, Université Paris 13, Sorbonne Paris Cite, Bobigny, France. ${ }^{3}$ Dept of Radiology, APHP, Avicenne Hospital, Bobigny, France. ${ }^{4}$ Dept of Cellular Biology, INSERM U1131, APHP, Saint Louis Hospital, Paris-Diderot University, Paris, France. ${ }^{5}$ These authors contributed equally.

Correspondence: Valérie Besnard, Universite Paris 13 Nord, EA 2363, 74 rue Marcel Cachin, Bobigny 93017, France. E-mail: valerie.besnard@univ-paris13.fr

Received: Aug 032018 | Accepted after revision: Sept 272018

Acknowledgements: The authors would like to thank J.F. Bernaudin (Dept of Respiratory Diseases, APHP, Avicenne Hospital, Bobigny, France) for his critical review and corrections of the manuscript.

Conflict of interest: C. Rotenberg has nothing to disclose. V. Besnard has nothing to disclose. P-Y. Brillet has nothing to disclose. S. Giraudier reports grants from Novartis. H. Nunes reports grants and personal fees for consultancy and research from Roche/Genentech and Boehringer Ingelheim, other (for investigator in clinical trials) from Sanofi and Gilead, outside the submitted work. D. Valeyre reports personal fees (scientific advisory board membership) from Boehringer Ingelheim and Roche, personal fees for lecturing from AstraZeneca, and personal fees for travel and accommodation from Boehringer Ingelheim and Roche, outside the submitted work.

\section{References}

1 Baughman RP, Nagai S, Balter M, et al. Defining the clinical outcome status (COS) in sarcoidosis: results of WASOG Task Force. Sarcoidosis Vasc Diffuse Lung Dis 2011; 28: 56-64. 
2 Mañá J, Rubio-Rivas M, Villalba N, et al. Multidisciplinary approach and long-term follow-up in a series of 640 consecutive patients with sarcoidosis: Cohort study of a 40 -year clinical experience at a tertiary referral center in Barcelona, Spain. Medicine 2017; 96: e7595.

3 Quintás-Cardama A, Vaddi K, Liu P, et al. Preclinical characterization of the selective JAK1/2 inhibitor INCB018424: therapeutic implications for the treatment of myeloproliferative neoplasms. Blood 2010; 115: 3109-3117.

4 O'Shea JJ, Plenge R. JAK and STAT signaling molecules in immunoregulation and immune-mediated disease. Immunity 2012; 36: 542-550.

5 Waters MJ, Brooks AJ. JAK2 activation by growth hormone and other cytokines. Biochem J 2015; 466: 1-11.

6 Sharma SM, Choi D, Planck SR, et al. Insights in to the pathogenesis of axial spondyloarthropathy based on gene expression profiles. Arthritis Res Ther 2009; 11: R168.

7 Muro S, Taha R, Tsicopoulos A, et al. Expression of IL-15 in inflammatory pulmonary diseases. J Allergy Clin Immunol 2001; 108: 970-975.

8 Kim HS, Choi D, Lim LL, et al. Association of interleukin 23 receptor gene with sarcoidosis. Dis Markers 2011; 31: 17-24.

9 Zhou T, Zhang W, Sweiss NJ, et al. Peripheral blood gene expression as a novel genomic biomarker in complicated sarcoidosis. PLoS ONE 2012; 7: e44818.

10 Zhou T, Casanova N, Pouladi N, et al. Identification of Jak-STAT signaling involvement in sarcoidosis severity via a novel microRNA-regulated peripheral blood mononuclear cell gene signature. Sci Rep 2017; 7: 4237.

11 Verstovsek S, Kantarjian H, Mesa RA, et al. Safety and efficacy of INCB018424, a JAK1 and JAK2 inhibitor, in myelofibrosis. N Engl J Med 2010; 363: 1117-1127.

12 Verstovsek S, Passamonti F, Rambaldi A, et al. A phase 2 study of ruxolitinib, an oral JAK1 and JAK2 inhibitor, in patients with advanced polycythemia vera who are refractory or intolerant to hydroxyurea. Cancer 2014; 120: 513-520.

13 Verstovsek S, Mesa RA, Gotlib J, et al. A double-blind, placebo-controlled trial of ruxolitinib for myelofibrosis. N Engl J Med 2012; 366: 799-807.

14 Weinacht KG, Charbonnier LM, Alroqi F, et al. Ruxolitinib reverses dysregulated $\mathrm{T}$ helper cell responses and controls autoimmunity caused by a novel signal transducer and activator of transcription 1 (STAT1) gainoffunction mutation. J Allergy Clin Immunol 2017; 139: 1629-1640.

15 Klaeschen AS, Wolf D, Brossart P, et al. JAK inhibitor ruxolitinib inhibits the expression of cytokines characteristic of cutaneous lupus erythematosus. J Exp Dermatol 2017; 26: 728-730.

16 Stöcklin E, Wissler M, Gouilleux F, et al. Functional interactions between Stat5 and the glucocorticoid receptor. Nature 1996; 383: 726-728.

17 Goleva E, Kisich KO, Leung DY. A role for STAT5 in the pathogenesis of IL-2-induced glucocorticoid resistance. J Immunol 2002; 169: 5934-5940.

18 Delgado-Martin C, Meyer LK, Huang BJ, et al. JAK/STAT pathway inhibition overcomes IL7-induced glucocorticoid resistance in a subset of human T-cell acute lymphoblastic leukemias. Leukemia 2017; 31: 2568-2576. 